\title{
Association of Fasting Glucose Level with Neutrophil-Lymphocyte Ratio Compared to Leukocyte Count and Serum C-Reactive Protein
}

\author{
Jin-Kyu Kim, Ah-Young Lee, Jee-Hyun Kang*, Byung-Yeon Yu, Seong-Ju Kim \\ Department of Family Medicine, Konyang University Hospital, Daejeon, Korea
}

\begin{abstract}
Background: The neutrophil-lymphocyte ratio (NLR) is often used as an inflammatory marker in chronic diseases such as cancer or cardiovascular diseases. However, there are few studies about the association between the NLR and diabetes mellitus (DM) or impaired fasting glucose (IFG) patients in Korea. This study investigated the association between the fasting plasma glucose (FPG) level and NLR in Koreans.

Methods: This cross-sectional retrospective study included 3,219 healthy subjects who visited Konyang University Hospital in South Korea for regular health examinations. Participants with a history of insulin administration, antidiabetic drugs, anti-inflammatory drugs, or underlying diseases related to inflammation were excluded.

Results: All statistical evaluation was performed by dividing participants into males and females. Based on FPG levels, the subjects were classified into three groups, with normal fasting glucose ( $\mathrm{n}=1,969), \operatorname{IFG}(\mathrm{n}=1,138)$, and DM $(\mathrm{n}=122)$. The NLR had no significant mean differences among these groups for both sexes. Multiple linear regression analysis between FPG level and NLR showed an independent and significantly negative association ( $\beta \pm$ standard error, $-0.67 \pm 0.24 ; \mathrm{P}=0.006$ ) in normal subjects after adjustment. $\log$ (serum C-reactive protein [S-CRP]) showed an independently and significantly positive association with FPG in male IFG/DM patients. Total leukocyte (white blood cell [WBC]) showed an independently and significantly positive association with FPG in female IFG/ DM patients.

Conclusion: In normal subjects, NLR shows an independently and significantly negative association with FPG. In IFG/DM patients, NLR was not significantly related to FPG. WBC count in female patients and S-CRP level in male patients were significantly positively associated with FPG only in IFG/DM.
\end{abstract}

Keywords: Neutrophils; Lymphocytes; Glucose; Diabetes Mellitus; Inflammation; Korean

Received: March 2, 2017, Revised: June 22, 2017, Accepted: July 11, 2017

*Corresponding Author: Jee-Hyun Kang https://orcid.org/0000-0003-4416-8895

Tel: +82-42-600-9240, Fax: +82-42-600-9095, E-mail: jkang@kyuh.ac.kr 


\section{INTRODUCTION}

Type 2 diabetes mellitus (DM) is an epidemic in Korea. About 4.8 million (13.7\%) Korean adults aged 30 years or older, had DM and 8.3 million $(24.8 \%)$ Korean adults had impaired fasting glucose (IFG) in 2014. ${ }^{1)}$

Insulin resistance is a metabolic disturbance that may result in central obesity and type $2 \mathrm{DM}$, as well as cardiovascular disease. ${ }^{2)}$ Several studies have confirmed that insulin resistance is associated with a chronic inflammatory state in which immunological changes play a key role in the initiation of type $2 \mathrm{DM}^{3-5)}$

Systemic inflammation is characterized by increased neutrophil and decreased lymphocyte counts in chronic diseases such as cancer and cardiovascular disease. ${ }^{6-8)}$ The neutrophil-lymphocyte ratio (NLR) is a laboratory test that does not have to be performed in the fasting state, and is a readily available inflammatory marker used to evaluate the severity of chronic diseases. ${ }^{9)}$ Although insulin resistance is closely related to chronic inflammatory status, ${ }^{10)}$ few studies have evaluated the relationship between the NLR and fasting plasma glucose (FPG) level or insulin resistance in Korea. Therefore, we assessed the association of FPG with inflammation based on the NLR, compared to other inflammatory markers such as serum C-reactive protein (S-CRP) and white blood cell (WBC) count. ${ }^{11)}$

\section{METHODS}

\section{Study Population and Design}

This retrospective cross-sectional study included 20,320 individuals that underwent health examinations at Konyang University Hospital in Daejeon, South Korea between June 1, 2014, and May 31, 2016. Medical records for 4,107 of these subjects were available for the assessment of chronic inflammatory status based on the levels of FPG, SCRP and insulin, as well as WBC.

We excluded subjects who were taking anti-diabetic drugs or injected insulin. Patients with hepatitis B virus (surface antigen positive) or (hepatitis C virus-RNA $>15 \mathrm{IU} / \mathrm{mL}$ or 40 copies $/ \mathrm{mL}$ ) infection, rheumatologic diseases (psoriasis, ankylosing spondylitis, or rheumatoid arthritis), asthma, and other forms of arthritis were also excluded, as well as examinees who were taking anti-allergic drugs, and regularly took steroids. Foreigners (regarding nationality and ethnicity) were also excluded, including those of Russian, African, American, Indian, Vietnamese, Chinese, and Japanese descent. Subjects whose WBC $>10,000$ were excluded by assuming acute inflammation. We did not exclude subjects with hypertension, hyperthyroidism, hypothyroidism, dyslipidemia, and depression. Based on the above exclusion criteria, a total of 3,219 subjects were selected for this study.

\section{Definitions}

DM and IFG were diagnosed based on the 2003 American Diabetes Association Expert Committee on the Diagnosis and Classification of Diabetes Mellitus using fasting glucose levels (DM: FPG $\geq 126 \mathrm{mg} / \mathrm{dL}$
[7.0 mmol/L] and IFG: FPG $\geq 100 \mathrm{mg} / \mathrm{dL}[5.6 \mathrm{mmol} / \mathrm{L}]$ to $<126 \mathrm{mg} / \mathrm{dL}$ $[6.9 \mathrm{mmol} / \mathrm{L}]){ }^{12)}$ The homeostatic model assessment of insulin resistance (HOMA-IR) index was calculated from fasting glucose and insulin levels at the visit using the following equation: ${ }^{13)}$ (fasting glucose $[\mathrm{mg} / \mathrm{dL}] \times$ fasting insulin $[\mu \mathrm{IU} / \mathrm{mL}]) \div 405$. The homeostatic model assessment of beta-cell function (HOMA-beta), which is used to identify pancreatic beta cell dysfunction, was calculated using the following equation: $^{(4)}$ (360×fasting insulin $\left.[\mu \mathrm{IU} / \mathrm{mL}]\right) \div$ (fasting glucose $[\mathrm{mg} / \mathrm{dL}]$ 63) (\%).

Participants' history of underlying disease, drug administration, alcohol intake, smoking, and exercise was collected using a subjectcompleted questionnaire saved in the medical record. Hypertension was diagnosed as systolic blood pressure $\geq 140 \mathrm{~mm} \mathrm{Hg}$, diastolic blood pressure $\geq 90 \mathrm{~mm} \mathrm{Hg}$, or the use of antihypertensive drugs. Smoking was defined as a participant smoking more than 100 cigarettes in his/ her whole lifetime. Alcohol intake was defined as the consumption of $>14 \mathrm{~g} / \mathrm{d}$. Exercise was defined as regular physical activity summing to at least 2 hours per week.

\section{Anthropometric Measurements}

Weight and height were measured using an automated machine (HM170; Fanics, Seoul, Korea) and bioelectrical impedance analyzer (Inbody 720; Biospace, Seoul, Korea) with subjects standing on a firm horizontal surface. Body mass index (BMI) was calculated using the formula: weight $(\mathrm{kg}) \div\left(\right.$ height $\left.{ }^{2}\right)\left(\mathrm{m}^{2}\right)$. Blood pressure was recorded to the nearest $2 \mathrm{~mm} \mathrm{Hg}$ from the right arm with the subject in a seated position (BPBIO320; Biospace, Cheonan, Korea). Two readings were taken 5 minutes apart, and the mean was recorded as the blood pressure. Waist circumference was measured using a tape passed through bilateral midpoints of a vertical line between the lowest rib and anterior superior iliac spine during mid-exhalation.

\section{Biochemical Parameters and Leukocyte Count}

Venous blood was drawn from the antecubital vein after fasting for at least 9 hours. WBC with differential count was measured using an LH780 analyzer (Beckman Coulter, Brea, CA, USA). S-CRP, total cholesterol, high-density lipoprotein cholesterol (HDL-C), low-density lipoprotein cholesterol (LDL-C), triacylglycerol (TG), FPG, protein, albumin, alanine transaminase (ALT), and aspartate transaminase (AST) levels were measured using an AU-5800 analyzer (Beckman Coulter, Shizuoka, Japan). Glycated hemoglobin (HbAlc) was measured using an ADAMS A1c HA-8180 machine (Arkray, Shiga, Japan). Insulin was measured using an RIA-mat 280 machine (STRATEC, Birkenfeld, Germany).

\section{Statistical Analysis}

Data for continuous biochemical skewed parameters (S-CRP, insulin, HOMA-IR, HOMA-beta, AST, ALT, and TG) were expressed as median (interquartile range) and the other continuous biochemical parameters were shown as mean \pm standard deviation (SD). By the law of large numbers, we assumed a normal distribution of values except for 
skewed variables. Skewness was tested by Q-Q plot in the R statistics program (http://www.r-project.org). Categorical values were expressed as numbers and percentages (\%). Wilcoxon rank-sum tests were used for skewed variables and t-tests were used non-skewed variables in Table 1. Since there were significant differences between males and females in many parameters, we needed to divide all subjects by sex to show demographic variables. Chi-square tests were used to compare categorical variables.

Kruskal-Wallis test was used for skewed variables and one-way analysis of variance test was used for non-skewed variables to assess whether there were differences in the median or mean difference among normal subjects, IFG, and DM patients in each gender category
(Table 2).

Spearman's correlation analysis was performed for skewed variables and Pearson's correlation analysis was done for non-skewed variables in Table 3. We merged IFG and DM patients into a patient group. Subgroup correlation analysis of normal and IFG/DM group was performed.

Multiple linear regression analysis was performed to investigate the relationship between FPG and inflammatory markers, such as NLR, WBC, and Log(S-CRP) (Table 4). To compare NLR, WBC, and Log(S$\mathrm{CRP}$ ), we used these variables as independent parameters and FPG as the dependent variable. We analyzed these data stratified according to the independent variables. In order to avoid multicollinearity, we as-

Table 1. Demographic and laboratory data classified according to sex

\begin{tabular}{|c|c|c|c|c|}
\hline Variable & Total $(\mathrm{N}=3,219)$ & Male $(\mathrm{N}=1,710)$ & Female $(\mathrm{N}=1,509)$ & P-value $e^{*, t}$ \\
\hline Age (y) & $48.81 \pm 11.68$ & $49.46 \pm 11.42$ & $48.07 \pm 11.93$ & $<0.001$ \\
\hline Waist circumference (cm) & $79.33 \pm 9.24$ & $83.38 \pm 8.02$ & $74.74 \pm 8.35$ & $<0.001$ \\
\hline Height (cm) & $164.77 \pm 8.98$ & $170.9 \pm 6.31$ & $157.82 \pm 6$ & $<0.001$ \\
\hline Weight (kg) & $65.68 \pm 12.59$ & $72.68 \pm 11.33$ & $57.76 \pm 8.62$ & $<0.001$ \\
\hline Body mass index $\left(\mathrm{kg} / \mathrm{m}^{2}\right)$ & $24.07 \pm 3.37$ & $24.83 \pm 3.23$ & $23.21 \pm 3.32$ & $<0.001$ \\
\hline Systolic blood pressure (mm Hg) & $112.95 \pm 14.15$ & $116 \pm 13.07$ & $109.51 \pm 14.53$ & $<0.001$ \\
\hline Diastolic blood pressure (mm Hg) & $73.88 \pm 9.31$ & $76.21 \pm 8.56$ & $71.23 \pm 9.42$ & $<0.001$ \\
\hline White blood cell $\left(\times 10^{3} / \mu \mathrm{L}\right)$ & $6.05 \pm 1.47$ & $6.43 \pm 1.45$ & $5.63 \pm 1.37$ & $<0.001$ \\
\hline Neutrophils (\%) & $53.9 \pm 8.61$ & $53.8 \pm 8.41$ & $54.01 \pm 8.84$ & 0.567 \\
\hline Lymphocytes (\%) & $35.62 \pm 7.86$ & $35 \pm 7.57$ & $36.32 \pm 8.11$ & 0.838 \\
\hline Monocytes (\%) & $6.95 \pm 1.79$ & $7.23 \pm 1.82$ & $6.63 \pm 1.7$ & 0.235 \\
\hline Neutrophil-lymphocyte ratio & $1.66 \pm 0.73$ & $1.68 \pm 0.73$ & $1.64 \pm 0.73$ & 0.600 \\
\hline Serum C-reactive protein $(\mathrm{mg} / \mathrm{dL})^{\ddagger}$ & $0.06(\mathrm{IQR}, 0.09)$ & $0.06(\mathrm{IQR}, 0.11)$ & $0.05(\mathrm{IQR}, 0.08)$ & $<0.001$ \\
\hline Fasting plasma glucose (mg/dL) & $98.97 \pm 15.3$ & $102.09 \pm 17.15$ & $95.43 \pm 11.95$ & $<0.001$ \\
\hline Glycated hemoglobin (\%) & $5.63 \pm 0.49$ & $5.67 \pm 0.56$ & $5.6 \pm 0.39$ & $<0.001$ \\
\hline Insulin $(\mu \mid \mathrm{U} / \mathrm{mL})^{\ddagger}$ & $3.62(\mathrm{IQR}, 3.71)$ & $3.61(\mathrm{QQR}, 3.81)$ & $3.62(\mathrm{IQR}, 3.6)$ & $<0.001$ \\
\hline HOMA-insulin resistance ${ }^{\ddagger}$ & $0.86(\mathrm{IQR}, 0.97)$ & $0.89(\mathrm{IQR}, 1.04)$ & $0.85(\mathrm{IQR}, 0.91)$ & $<0.001$ \\
\hline HOMA-beta-cell ${ }^{\ddagger}$ & $37.92($ (IQR, 38.97) & $35.27(\mathrm{IQR}, 37.3)$ & 41.78 (IQR, 40.97) & $<0.001$ \\
\hline Albumin (g/dL) & $4.5 \pm 0.26$ & $4.56 \pm 0.26$ & $4.43 \pm 0.25$ & $<0.001$ \\
\hline Protein (g/dL) & $7.26 \pm 0.4$ & $7.29 \pm 0.4$ & $7.24 \pm 0.39$ & $<0.001$ \\
\hline Alanine transaminase $(\mathrm{IU} / \mathrm{L})^{\ddagger}$ & $25(\mathrm{IQR}, 17)$ & $28(\mathrm{IQR}, 21)$ & $23(\mathrm{IQR}, 11)$ & $<0.001$ \\
\hline Aspartate transaminase $(\mathrm{IU} / \mathrm{L})^{\ddagger}$ & $22(\mathrm{IQR}, 12)$ & $28(\mathrm{IQR}, 13)$ & $17(\mathrm{IQR}, 10)$ & $<0.001$ \\
\hline Total cholesterol (mg/dL) & $199.06 \pm 37.13$ & $200.03 \pm 37.89$ & $197.96 \pm 36.24$ & $<0.001$ \\
\hline Low-density lipoprotein cholesterol (mg/dL) & $137.41 \pm 32.51$ & $140.37 \pm 32.48$ & $134.05 \pm 32.22$ & $<0.001$ \\
\hline High-density lipoprotein cholesterol (mg/dL) & $58.05 \pm 13.76$ & $54.23 \pm 12.46$ & $62.37 \pm 13.9$ & $<0.001$ \\
\hline Triacylglycerol $(\mathrm{mg} / \mathrm{mL})^{\ddagger}$ & $111.1($ IQR, 86.6) & 131.05 (IQR, 101.33) & $94.8(\mathrm{IQR}, 64.7)$ & $<0.001$ \\
\hline Alcohols & & & & $<0.001$ \\
\hline Yes & 2488 (77.1) & $1,511(88.4)$ & $972(64.4)$ & \\
\hline No & $741(22.9)$ & $199(11.6)$ & $537(35.6)$ & \\
\hline Smoke & & & & $<0.001$ \\
\hline Yes & 1,416 (43.8) & $1,276(74.6)$ & $135(8.9)$ & \\
\hline No & $1,813(56.2)$ & $434(25.4)$ & $1,374(91.1)$ & \\
\hline Hypertension & & & & $<0.001$ \\
\hline Yes & $530(16.4)$ & 335 (19.6) & $194(12.9)$ & \\
\hline No & 2,699 (83.6) & $1,375(80.4)$ & $1,315(87.1)$ & \\
\hline Exercise & & & & $<0.001$ \\
\hline Yes & $1,186(36.7)$ & $675(39.5)$ & 507 (33.6) & \\
\hline No & $2,043(63.3)$ & $1,035(60.5)$ & $1,002(66.4)$ & \\
\hline
\end{tabular}

Values are presented as mean \pm standard deviation for continuous variables, number (\%) for categorical variables, or median (IQR) for known skewed variables.

IQR, interquartile range; HOMA, homeostatic model assessment.

${ }^{*}$ Calculated by Wilcoxon rank-sum test for known skewed variable ${ }^{\ddagger}$ and calculated by $t$-test for continuous variables and chi-square test for categorical variables. ${ }^{\dagger} \mathrm{P}<0.05$ was accepted as the level of significance. ${ }^{\S}$ Alcohol intake defined as the consumption of $>14 \mathrm{~g} / \mathrm{d}$ for both sexes. 
लำ

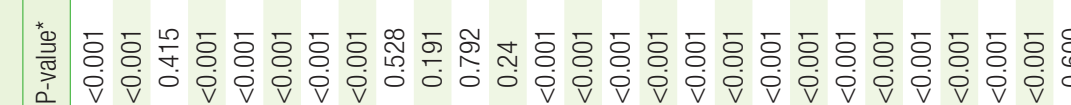

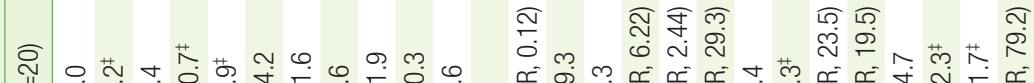
II

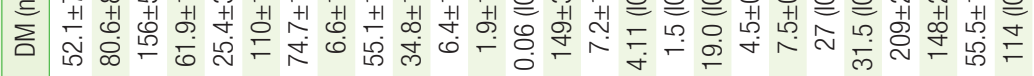

$\stackrel{\text { II }}{z}$

峁

焉

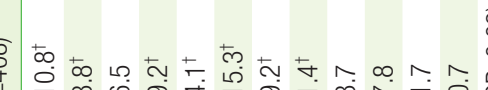

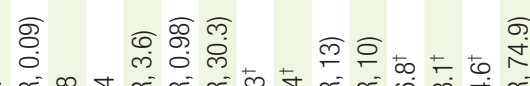

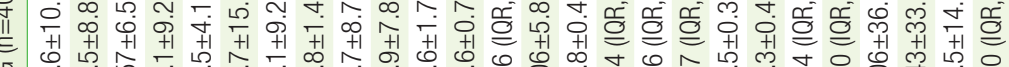

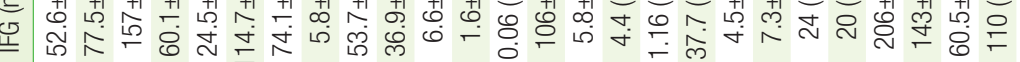

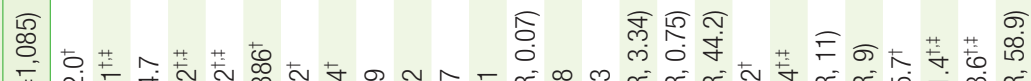

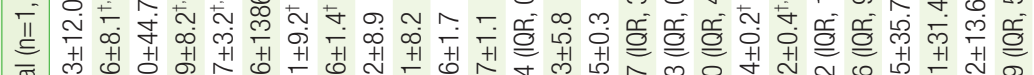

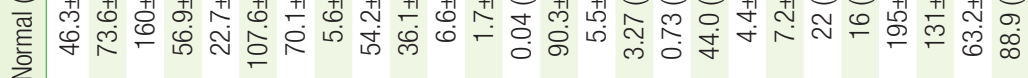

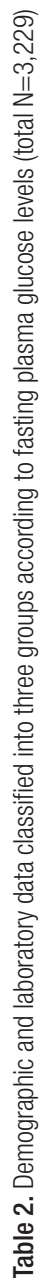

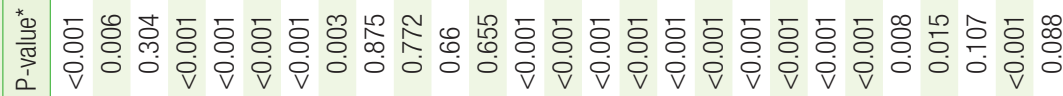

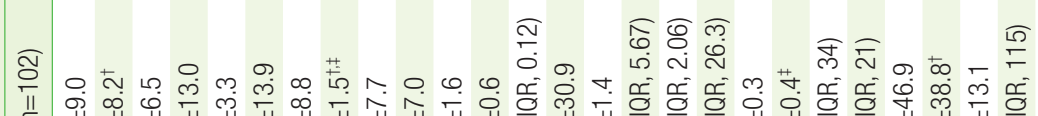

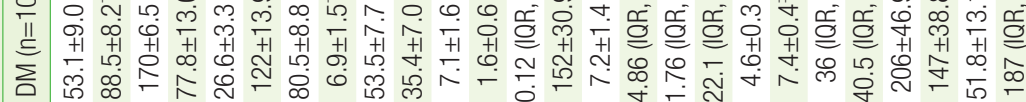
(6)

$\div \stackrel{\circ}{\circ}$

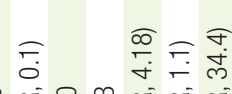

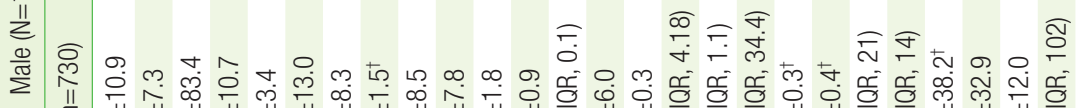

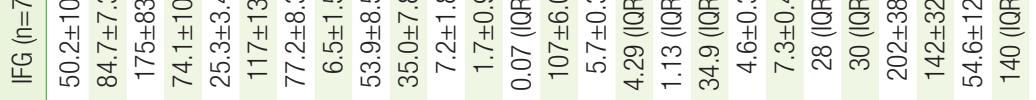

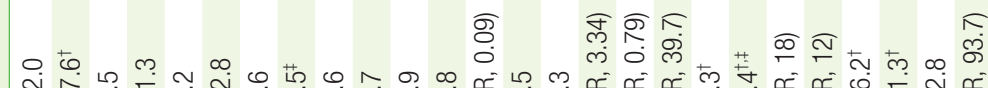

萨 光 产

कุ बं. 品六 总 辛

דִ

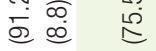

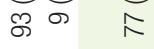
我 $\sqrt{5}$ $\stackrel{2}{\sim}$

₹.

迎 สฺ 过

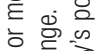

잉

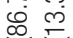
$\stackrel{\infty}{\rightleftharpoons}$

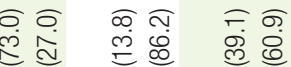

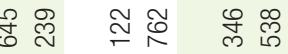


Table 3. Correlation between neutrophil-lymphocyte ratio and other factors divided into normal fasting glucose and IFG/DM

\begin{tabular}{|c|c|c|c|c|c|c|c|c|}
\hline \multirow{3}{*}{ Variable } & \multicolumn{4}{|c|}{ Male $(\mathrm{N}=1,716)$} & \multicolumn{4}{|c|}{ Female $(\mathrm{N}=1,513)$} \\
\hline & \multicolumn{2}{|c|}{ Normal fasting glucose $(n=884)$} & \multicolumn{2}{|c|}{ IFG/DM (n=832) } & \multicolumn{2}{|c|}{ Normal fasting glucose $(n=1,085)$} & \multicolumn{2}{|c|}{ IFG/DM (n=428) } \\
\hline & r & P-value & r & $P$-value & r & P-value & r & P-value \\
\hline Age $(y)$ & 0.09 & 0.009 & 0.15 & $<0.001$ & -0.15 & $<0.001$ & -0.1 & 0.049 \\
\hline Waist circumference (cm) & -0.05 & 0.156 & -0.1 & 0.005 & -0.03 & 0.337 & -0.02 & 0.73 \\
\hline Body mass index $\left(\mathrm{kg} / \mathrm{m}^{2}\right)$ & -0.09 & 0.008 & -0.1 & 0.004 & -0.03 & 0.303 & -0.007 & 0.881 \\
\hline White blood cell $\left(\times 10^{3} / \mu \mathrm{L}\right)$ & 0.35 & $<0.001$ & 0.27 & $<0.001$ & 0.25 & $<0.001$ & 0.42 & $<0.001$ \\
\hline Serum C-reactive protein $(\mathrm{mg} / \mathrm{dL})^{*}$ & 0.24 & $<0.001$ & 0.11 & 0.002 & 0.19 & $<0.001$ & 0.19 & $<0.001$ \\
\hline $\mathrm{FPG}(\mathrm{mg} / \mathrm{dL})$ & -0.15 & $<0.001$ & -0.004 & 0.916 & -0.07 & 0.028 & 0.07 & 0.125 \\
\hline FPG-neutrophil & -0.10 & 0.005 & 0.01 & 0.704 & -0.1 & 0.001 & 0.07 & 0.133 \\
\hline FPG-lymphocyte & 0.11 & 0.001 & -0.03 & 0.473 & 0.1 & $<0.001$ & -0.1 & 0.052 \\
\hline Glycated hemoglobin (\%) & -0.02 & 0.586 & -0.002 & 0.943 & -0.13 & $<0.001$ & -0.04 & 0.417 \\
\hline Insulin $(\mu \mathrm{lU} / \mathrm{mL})^{*}$ & 0.003 & 0.936 & 0.01 & 0.789 & 0.08 & 0.007 & 0.15 & 0.002 \\
\hline HOMA-insulin resistance ${ }^{*}$ & -0.004 & 0.896 & 0.01 & 0.817 & 0.08 & 0.012 & 0.15 & 0.001 \\
\hline HOMA-beta-cell ${ }^{*}$ & 0.04 & 0.248 & 0.01 & 0.721 & 0.1 & 0.001 & 0.13 & 0.008 \\
\hline Low-density lipoprotein cholesterol (mg/dL) & -0.06 & 0.085 & -0.14 & $<0.001$ & -0.16 & $<0.001$ & -0.11 & 0.022 \\
\hline High-density lipoprotein cholesterol (mg/dL) & -0.01 & 0.756 & -0.02 & 0.615 & -0.08 & 0.009 & 0.02 & 0.643 \\
\hline Triacylglycerol $(\mathrm{mg} / \mathrm{mL})^{*}$ & -0.05 & 0.175 & -0.03 & 0.38 & 0.02 & 0.515 & 0.03 & 0.504 \\
\hline
\end{tabular}

IFG/DM, impaired fasting glucose/diabetes mellitus; FPG, fasting plasma glucose; HOMA, homeostatic model assessment.

*Skewed variables were analyzed by Spearman's correlation analysis. And the other variables were calculated by Pearson's correlation analysis.

Table 4. Multiple linear regression analysis of factors independently associated with fasting plasma glucose

\begin{tabular}{|c|c|c|c|c|c|c|c|c|c|c|c|c|}
\hline \multirow{4}{*}{ Variable } & \multicolumn{6}{|c|}{ Male $(\mathrm{N}=1,716)$} & \multicolumn{6}{|c|}{ Female $(\mathrm{N}=1,513)$} \\
\hline & \multicolumn{3}{|c|}{ Normal $(n=884)$} & \multicolumn{3}{|c|}{ IFG/DM (n=832) } & \multicolumn{3}{|c|}{ Normal $(n=1,085)$} & \multicolumn{3}{|c|}{ IFG/DM (n=428) } \\
\hline & $\beta \pm \mathrm{SE}$ & \multirow{2}{*}{ P-value* } & \multirow{2}{*}{$\begin{array}{c}\text { Adjusted } \\
\mathrm{R}^{2}\end{array}$} & \multirow{2}{*}{$\begin{array}{c}\beta \pm S E \\
\text { Log(S-CRP) }\end{array}$} & \multirow{2}{*}{ P-value* } & \multirow{2}{*}{$\begin{array}{c}\text { Adjusted } \\
\mathrm{R}^{2}\end{array}$} & \multirow{2}{*}{$\begin{array}{c}\beta \pm S E \\
N L R\end{array}$} & \multirow{2}{*}{ P-value } & \multirow{2}{*}{$\begin{array}{c}\text { Adjusted } \\
\mathrm{R}^{2}\end{array}$} & $\beta \pm \mathrm{SE}$ & \multirow{2}{*}{ P-value } & \multirow{2}{*}{$\begin{array}{c}\text { Adjusted } \\
\mathrm{R}^{2}\end{array}$} \\
\hline & NLR & & & & & & & & & WBC & & \\
\hline Model 1 & $-0.86 \pm 0.23$ & $<0.001$ & 0.014 & $2.8 \pm 0.66$ & $<0.001$ & 0.02 & $-0.95 \pm 0.24$ & $<0.001$ & 0.013 & $1.11 \pm 0.33$ & $<0.001$ & 0.024 \\
\hline \multicolumn{13}{|l|}{ Model 2} \\
\hline NLR & $-0.73 \pm 0.25$ & $<0.001$ & 0.047 & $-0.94 \pm 0.98$ & 0.336 & 0.031 & $-0.89 \pm 0.26$ & $<0.001$ & 0.072 & $0.68 \pm 0.75$ & 0.368 & 0.026 \\
\hline WBC & $-0.004 \pm 0.14$ & 0.223 & & $0.75 \pm 0.49$ & 0.126 & & $0.20 \pm 0.15$ & 0.162 & & $0.83 \pm 0.37$ & 0.029 & \\
\hline Log(S-CRP) & $-0.05 \pm 0.18$ & 0.464 & & $2.25 \pm 0.71$ & 0.002 & & $0.12 \pm 0.18$ & 0.527 & & $-0.005 \pm 0.46$ & 0.991 & \\
\hline \multicolumn{13}{|l|}{ Model 3} \\
\hline NLR & $-0.67 \pm 0.24$ & 0.006 & 0.136 & $-0.16 \pm 0.95$ & 0.868 & 0.1 & $-0.77 \pm 0.26$ & 0.003 & 0.163 & $1.13 \pm 0.74$ & 0.125 & 0.09 \\
\hline WBC & $-0.21 \pm 0.14$ & 0.121 & & $0.21 \pm 0.49$ & 0.663 & & $-0.04 \pm 0.14$ & 0.77 & & $1.03 \pm 0.38$ & 0.006 & \\
\hline Log(S-CRP) & $0.003 \pm 0.18$ & 0.859 & & $2.23 \pm 0.69$ & 0.001 & & $0.12 \pm 0.17$ & 0.5 & & $-0.07 \pm 0.46$ & 0.884 & \\
\hline Log(HOMA) & $0.79 \pm 0.14$ & $<0.001$ & $(-\mathrm{IR})$ & $-4.39 \pm 0.61$ & $<0.001$ & (-Beta) & $1.45 \pm 0.16$ & $<0.001$ & $(-\mathrm{IR})$ & $-2.75 \pm 0.54$ & $<0.001$ & (-Beta) \\
\hline \multicolumn{13}{|l|}{ Model 4} \\
\hline NLR & $-0.66 \pm 0.25$ & 0.008 & 0.122 & $-0.65 \pm 0.98$ & 0.51 & 0.042 & $-0.76 \pm 0.26$ & 0.003 & 0.138 & $0.95 \pm 0.76$ & 0.212 & 0.034 \\
\hline WBC & $-0.22 \pm 0.14$ & 0.11 & & $0.26 \pm 0.51$ & 0.612 & & $-0.04 \pm 0.15$ & 0.776 & & $0.89 \pm 0.39$ & 0.022 & \\
\hline Log(S-CRP) & $0.01 \pm 0.18$ & 0.952 & & $2.14 \pm 0.71$ & 0.003 & & $0.11 \pm 0.18$ & 0.543 & & $-0.16 \pm 0.47$ & 0.733 & \\
\hline Log(insulin) & $0.60 \pm 0.14$ & $<0.001$ & & $-0.08 \pm 0.64$ & 0.900 & & $1.15 \pm 0.16$ & $<0.001$ & & $-0.50 \pm 0.57$ & 0.379 & \\
\hline
\end{tabular}

Model 1: unadjusted; model 2: adjusted for age and BMI; model 3: adjusted for age, BMI, and lipid profile ${ }^{\dagger}$; model 4: adjusted for age, BMI, and lipid profile ${ }^{\dagger}$.

IFG/DM, impaired fasting glucose/diabetes mellitus; $\beta \pm S E$, standardized regression coefficient \pm standard error; $R^{2}$, explanatory power of variables on fasting plasma glucose; NLR, neutrophil-Iymphocyte ratio; Log, natural logarithm; S-CRP, serum C-reactive protein; WBC, white blood cell; HOMA-IR, homeostatic model assessment-insulin resistance; HOMA-beta, homeostatic model assessment-beta-cell; BMl, body mass index.

*Calculated by multiple linear regression analysis. 'Lipid profile contains low-density lipoprotein cholesterol, high-density lipoprotein, and log(triacylglycerol) variables.

sessed variation inflation factor levels of independent variables to be lower than 10 in each model. In model 1, we analyzed the association between inflammatory markers and FPG using simple linear regression and expressed only significant independent variables among NLR, WBC, and Log(S-CRP). In models 2, 3, and 4, independent variables were added to the previous model to find out explanatory power of parameters. HOMA-IR and HOMA-Beta had significant independent relationship with FPG in each subgroup. We represented which HOMA variable was significant by writing nearby P-value, such as (-IR) or (-beta). Since the variables insulin, HOMA-IR and HOMA-Beta were multicollinear, they were not used as independent variables simultaneously. Although age, BMI, LDL-C, HDL-C, and Log(TG) were 
also used as independent variables in each model, we omitted these data for simplicity.

All analyses were performed using the R statistical package ver. 3.3.1 (http://www.r-project.org) with R Studio ver. 0.99 (Cygnus Inc., Redwood City, CA, USA), which are open source software that follow the Lesser General Public License ver. 2.1 (http://www.gnu.org/licenses/ lgpl.html). P-values $<0.05$ were considered statistically significant.

\section{RESULTS}

\section{Baseline Characteristics of the Demographic and Laboratory Data}

The baseline characteristics of the participants are listed in Table 1. The mean \pm SD of age among males was $49.5 \pm 11.4$ years, and $48.1 \pm 11.9$ years for females. Most parameters significantly differed in median or mean between males and females. The means of neutrophil, lymphocyte, monocyte, and NLR were not significantly different between both sexes. Most variables that affect glucose metabolism or represent chronic inflammation were worse in males than females (higher: BMI, SBP, DBP, WBC, S-CRP, FPG, HbAlc, LDL-C, and TG; lower: HOMAbeta and HDL-C). Lifestyle variables (alcohol consumption, smoking, hypertension, and exercise rate) were also higher in males than females.

Table 2 presents, based on FPG levels, the examinees classified into three groups for each sex, respectively (male subjects: normal [n=884], IFG [ $n=730]$, and DM [n=102]; female subjects: normal [n=1,085], IFG $[\mathrm{n}=408]$, and DM [n=20]). NLR was not significantly different among the three groups (normal, IFG, and DM) for both sexes. There were also no significant differences in neutrophil and lymphocyte, themselves, among the three groups for both sexes. Inflammatory markers (S-CRP and WBC), glucose metabolism parameters (HbAlc, insulin, and HOMA-IR) and demographic parameters (age, BMI, SBP, DBP, and waist circumference) were higher in the IFG and DM groups than in the normal groups for both sexes. These parameters worsened with increasing FPG. All lipid profile variables among females were higher in the IFG and DM groups. Among the lifestyle parameters, only hypertension prevalence was significantly higher in IFG and DM group for both sexes.

\section{Correlation between Neutrophil-Lymphocyte Ratio and Other Factors with Subgroup Analysis for Both Sexes}

Table 3 shows the correlation between the NLR and other parameters by Spearman's or Pearson's correlation analysis. WBC and S-CRP were significantly positively correlated with NLR in all subgroups for both sexes. In the normal subgroup, FPG was significantly negatively correlated with NLR while the IFG/DM subgroup did not demonstrate any such correlation for both sexes. Neutrophils and lymphocytes, themselves, were significantly correlated with FPG with correspondence to negative correlation between NLR with FPG. In the IFG/DM patients group, there was no significant correlation between FPG and NLR, and neutrophils and lymphocyte for both sexes. We found significant cor- relation between NLR and HOMA, insulin, and LDL-C in female subjects for the normal and patient subgroups.

\section{Multiple Linear Regression Analysis of Fasting Plasma Glucose Associated with Neutrophil-Lymphocyte Ratio, White Blood Cell, and Log(Serum C-Reactive Protein)}

Both men and women were divided into two groups according to the fasting glucose level such as normal fasting glucose subjects group (FPG $<100 \mathrm{mg} / \mathrm{dL}$ ) and IFG/DM patients group (FPG $\geq 100 \mathrm{mg} / \mathrm{dL}$ ). We performed simple or multiple linear regressions in each subgroup, as described in Table 4. In model 1 of the simple linear regression results, FPG levels were significantly negatively associated with NLR in the normal subjects group for both sexes (male: $\beta \pm$ standard error [SE], $-0.86 \pm 0.23$; $\mathrm{P}<0.001$; female: $\beta \pm \mathrm{SE},-0.95 \pm 0.24 ; \mathrm{P}<0.001)$. WBC and Log(S-CRP) were not significantly associated with FPG in the normal subjects group. $\log (\mathrm{S}-\mathrm{CRP})$ was significantly positively associated with FPG in the male IFG/DM patients group $(\beta \pm S E, 2.8 \pm 0.66 ; \mathrm{P}<0.001)$. WBC was positively significantly associated with FPG in the female IFG/DM patients group $(\beta \pm S E, 1.11 \pm 0.33 ; \mathrm{P}<0.001)$.

In model 2, we compared the three inflammatory markers NLR, WBC, and $\log (\mathrm{S}-\mathrm{CRP})$ using multiple linear regression analysis after adjusting for age and BMI. In model 3, HOMA variables and lipid profile variables were adjusted additionally. In model 4, to figure out the effect of insulin on normal subjects, we substituted the variable HOMA with Log(insulin). In all models, both men and women who had normal FPG levels had significantly negative association between FPG and NLR. Log(S-CRP) was significantly positively associated with FPG only in the male IFG/DM group. WBC was significantly positively associated with FPG only in the female IFG/DM group. In model 3, particularly, $\log (\mathrm{HOMA}-\mathrm{IR})$ was significantly positively associated with FPG in the normal group for both sexes, while Log(HOMA-beta) was significantly negatively associated with FPG in the IFG/DM group for both sexes. Log(insulin) level was significantly positively associated with FPG level only in the normal fasting glucose group for both sexes in model 4 .

\section{DISCUSSION}

The results of this study suggested that there were significant differences in inflammatory markers among subgroups divided by sex and FPG state. NLR was not a reliable inflammatory marker in IFG/DM patients and was significantly negatively associated with FPG level only in the normal fasting glucose group for both sexes. We suggest that lymphocyte proliferation plays a key role in the effect of NLR on insulin secretion in normal subjects.

\section{Lymphocyte Proliferation with Increase in Glucose Level in Normal Subjects}

Otton et al. ${ }^{15)}$ argued that insulin level had a significantly positive relationship with lymphocyte proliferation in normal and diabetic rats. Insulin promoted a remarkable increase in lymphocyte proliferation 
(7.5-fold) in the normal rat group and this response was much lower (2.6-fold) in lymphocytes from diabetic rats. Our study suggested that subjects who had normal fasting glucose might preserve their insulin secretion function well. In normal subjects, elevated glucose level induced enough insulin secretion, and this affected the proliferation of lymphocytes more than subjects with lower glucose levels in the normal fasting glucose group. This suggestion was consistent with the result of this study, that is, the increase in lymphocytes when FPG and insulin increased in the normal fasting glucose group (Table 3 and model 4 of Table 4). On the other hand, another study reported that mitogen-induced lymphocyte proliferation was $19 \%-32 \%$ lower in obese (mean BMI, $33.2 \pm 6.5 \mathrm{~kg} / \mathrm{m}^{2}$ ) than non-obese subjects (mean BMI, $21.2 \pm 1.9 \mathrm{~kg} / \mathrm{m}^{2}$ ), when adjusted for T and B lymphocyte count. ${ }^{16)}$ Insulin is a well-known mitogenic hormone. ${ }^{17)}$ Insulin seemed to have an effect on lymphocyte proliferation, regardless of mitogenicity. Further investigation will be needed in this regard.

\section{Neutrophil Count Fluctuation}

An experimental study of mice fed a high-fat diet showed that neutrophil secretion of elastase resulted in insulin resistance. ${ }^{18)}$ Hyperglycemia inhibits the apoptosis of neutrophils and prolongs inflammation in diabetic mice. Decreased apoptosis of neutrophils is associated with impaired clearance of neutrophils by macrophages in diabetic mice. ${ }^{19)}$ Our study suggests that normal subjects seemed to have an intact ability of macrophages to clear neutrophils and stabilize their counts. This relationship in our study (Table 3), which was between neutrophils and FPG in the normal fasting glucose group was suggested due to the relative increase in lymphocyte count in response to an increased neutrophil count $(\mathrm{r}=-0.10, \mathrm{P}=0.005$ in males; $\mathrm{r}=-0.1$, $\mathrm{P}=0.001$ in females).

\section{NLR as an Inflammatory Marker}

NLR was not a more significant inflammatory marker than WBC and $\mathrm{S}-\mathrm{CRP}$ in IFG/DM patients group in our research. NLR had no statistically significant relationship with FPG in IFG/DM patients. FPG level is a criterion in diagnosing metabolic syndrome. Bahadir et al. ${ }^{20}$ showed NLR was not significantly different according to severity of metabolic syndrome. The leukocyte, neutrophil, and lymphocyte counts significantly increased with increasing degree of obesity and BMI without classification of normal or pre-diabetes/DM. In a Turkish study, there were no significant differences in NLR among subjects with euglycemia, IFG, and impaired glucose tolerance (IGT). ${ }^{21)}$ On the other hand, some studies revealed that high levels of lymphocyte apoptosis were triggered by the development of DM and hyperglycemia. ${ }^{22,23)}$ Shiny et al. ${ }^{24)}$ reported higher NLR and neutrophil levels, and lower lymphocyte counts in patients with DM and IGT compared to normal subjects who were mostly Indian. Another study showed that the higher the NLR, the higher the HbAlc level. ${ }^{25)}$

In the IFG/DM group of our study, NLR was not a reasonable biomarker and was not associated with FPG which represented severity of IFG/DM. NLR, neutrophil and lymphocyte levels were not signifi- cantly associated with FPG in IFG/DM patients of both sexes, respectively. The results of the linear regression of neutrophil and lymphocyte counts with FPG were omitted in Table 4. In the IFG/DM group, most of the other studies which showed that NLR had a positive association with FPG was only for individuals with metabolic syndrome or type 2 DM patients. ${ }^{24,25)}$ Few studies have evaluated the association between NLR and FPG in normal subjects. Other studies showed a significantly positive association between NLR and FPG in the patients group, while our results did not show such an association in the IFG/ DM group. Thus, we guess there could be a possible difference in underlying pathophysiology between the participants of this study and those of other studies. Ohn et al. ${ }^{26)}$ reported that in Korean or Oriental subjects, the impairment of beta-cell function has a more significant effect on the onset of DM than insufficient insulin sensitivity does. In a research of 3,059 Japanese subjects who regularly visited for medical check-ups, insufficient beta-cell function had a greater effect on the aggravation of diabetes than insulin resistance. ${ }^{27)}$ We suggest that the positively significant association between NLR and glucose level in other studies was due to genetic differences between Oriental and Western subjects. In the Western study mentioned previously, insulin resistance, obesity, and high BMI had a tendency to affect the onset of DM, while insufficient insulin secretion function tended to affect the pathophysiology of DM in Oriental or Korean subjects. Impairment of insulin secretion function was more predictive of incident DM than insulin sensitivity in Koreans. ${ }^{26)}$ Five genes (UBE2E2, ST6GAL1, GCK, $K C N J 11$, and $C 2 C D 4 A$ ) are known to be associated with pre-diabetes and DM according to the Beta Cell Gene Atlas. ${ }^{28)}$ Glucokinase plays a key role in insulin secretion because it senses the glucose concentration in pancreatic beta cells. A pathological mutation in the GCK gene induces monogenic diabetes of the young type 2 . This genetic variation has been associated with the impairment of glucose tolerance. ${ }^{29)}$ In addition, a combined meta-analysis for type $2 \mathrm{DM}$ revealed two new loci, CCDC63 and C12orf51 in the 12q24 region. These variants were significantly associated with FPG and HOMA-beta in Korean males with high-specificity. ${ }^{30)}$ In short, Oriental or Korean subjects had genetic features of fragility in insulin secretion function while insulin resistance in Western subjects was a more important feature in the onset of pre-diabetes or DM.

\section{Serum C-Reactive Protein and White Blood Cell in Impaired Fasting Glucose/Diabetes Mellitus Patients}

Bahadir et al. ${ }^{20)}$ revealed that WBC and CRP levels were higher in the more severe metabolic syndrome patients than normal non-diabetic subjects. Our study also showed that $\log (\mathrm{S}-\mathrm{CRP})$ was elevated with the increase in severity of IFG/DM in male patients and WBC was also increased in female IFG/DM patients. There were no differences in inflammatory markers according to gender.

\section{Limitations}

Several potential limitations should be considered. First, IFG and DM were diagnosed only by fasting glucose levels for clinical convenience 
in the general health examination center. To evaluate which inflammatory markers are effective in detail, further research is necessary regarding the association between NLR and HbAlc levels or glucose tolerance in Koreans. More specialized subgroup classification would also be needed. Second, the cross-sectional retrospective design prevented the identification of causality. Third, we investigated individuals without severe symptoms who were able to visit the health examination center of his/her own accord; this potential selection bias may have affected the results of this study.

\section{Conclusion}

The present study provides evidence that NLR has an independently and significantly negative relationship with FPG in normal subjects. As an inflammatory marker, NLR is not a reasonable biomarker in both normal and IFG/DM patient subgroups. S-CRP had an independently and significantly positive relationship with FPG in male IFG/DM patients. WBC had an independently and significantly positive relationship with FPG in male IFG/DM patients. S-CRP and WBC had no significant relationship with FPG in normal subjects. Research related to sexual differences in inflammatory markers could hardly be found. Further research would be needed. The finding of our study demonstrates that NLR is clinically significant as an inverse parameter of FPG level in normal subjects. S-CRP and WBC are clinically significant only in Korean IFG/DM patients.

\section{CONFLICT OF INTEREST}

No potential conflict of interest relevant to this article was reported.

\section{REFERENCES}

1. Korean Diabetes Association. Diabetes fact sheet in Korea 2013. Seoul: Korean Diabetes Association; 2013.

2. McKeigue PM, Shah B, Marmot MG. Relation of central obesity and insulin resistance with high diabetes prevalence and cardiovascular risk in South Asians. Lancet 1991;337:382-6.

3. Shoelson SE, Lee J, Goldfine AB. Inflammation and insulin resistance. J Clin Invest 2006;116:1793-801.

4. Bastard JP, Maachi M, Lagathu C, Kim MJ, Caron M, Vidal H, et al. Recent advances in the relationship between obesity, inflammation, and insulin resistance. Eur Cytokine Netw 2006;17:4-12.

5. Xu H, Barnes GT, Yang Q, Tan G, Yang D, Chou CJ, et al. Chronic inflammation in fat plays a crucial role in the development of obesity-related insulin resistance. J Clin Invest 2003;112:1821-30.

6. Imtiaz F, Shafique K, Mirza SS, Ayoob Z, Vart P, Rao S. Neutrophil lymphocyte ratio as a measure of systemic inflammation in prevalent chronic diseases in Asian population. Int Arch Med 2012;5:2.

7. Papa A, Emdin M, Passino C, Michelassi C, Battaglia D, Cocci F. Predictive value of elevated neutrophil-lymphocyte ratio on cardiac mortality in patients with stable coronary artery disease. Clin Chim Acta 2008;395:27-31.

8. Templeton AJ, Knox JJ, Lin X, Simantov R, Xie W, Lawrence N, et al. Change in neutrophil-to-lymphocyte ratio in response to targeted therapy for metastatic renal cell carcinoma as a prognosticator and biomarker of efficacy. Eur Urol 2016;70:358-64.

9. Arbel Y, Finkelstein A, Halkin A, Birati EY, Revivo M, Zuzut M, et al. Neutrophil/lymphocyte ratio is related to the severity of coronary artery disease and clinical outcome in patients undergoing angiography. Atherosclerosis 2012;225:456-60.

10. Rippe JM, Crossley S, Ringer R. Obesity as a chronic disease: modern medical and lifestyle management. J Am Diet Assoc 1998;98(10 Suppl 2):S9-15.

11. Kaplan M, Hamoud S, Tendler Y, Meilin E, Lazarovitch A, Nitecki S, et al. A significant correlation between C-reactive protein levels in blood monocytes derived macrophages versus content in carotid atherosclerotic lesions. J Inflamm (Lond) 2014;11:7.

12. Expert Committee on the Diagnosis and Classification of Diabetes Mellitus. Report of the expert committee on the diagnosis and classification of diabetes mellitus. Diabetes Care 2003;26 Suppl 1:S5-20.

13. Haffner SM, Miettinen H, Stern MP. The homeostasis model in the San Antonio Heart Study. Diabetes Care 1997;20:1087-92.

14. Matthews DR, Hosker JP, Rudenski AS, Naylor BA, Treacher DF, Turner RC. Homeostasis model assessment: insulin resistance and beta-cell function from fasting plasma glucose and insulin concentrations in man. Diabetologia 1985;28:412-9.

15. Otton R, Carvalho CR, Mendonça JR, Curi R. Low proliferation capacity of lymphocytes from alloxan-diabetic rats: involvement of high glucose and tyrosine phosphorylation of Shc and IRS-1. Life Sci 2002;71:2759-71.

16. Nieman DC, Henson DA, Nehlsen-Cannarella SL, Ekkens M, Utter AC, Butterworth DE, et al. Influence of obesity on immune function. J Am Diet Assoc 1999;99:294-9.

17. Bedinger DH, Adams SH. Metabolic, anabolic, and mitogenic insulin responses: a tissue-specific perspective for insulin receptor activators. Mol Cell Endocrinol 2015;415:143-56.

18. Talukdar S, Oh DY, Bandyopadhyay G, Li D, Xu J, McNelis J, et al. Neutrophils mediate insulin resistance in mice fed a high-fat diet through secreted elastase. Nat Med 2012;18:1407-12.

19. Hanses F, Park S, Rich J, Lee JC. Reduced neutrophil apoptosis in diabetic mice during staphylococcal infection leads to prolonged $\operatorname{Tnf} \alpha$ production and reduced neutrophil clearance. PLoS One 2011;6:e23633.

20. Bahadir A, Baltaci D, Turker Y, Turker Y, Iliev D, Ozturk S, et al. Is the neutrophil-to-lymphocyte ratio indicative of inflammatory state in patients with obesity and metabolic syndrome? Anatol J Cardiol 2015;15:816-22.

21. Aoyama-Sasabe S, Fukushima M, Xin X, Taniguchi A, Nakai Y, Mitsui $\mathrm{R}$, et al. Insulin secretory defect and insulin resistance in isolated impaired fasting glucose and isolated impaired glucose tolerance. J Diabetes Res 2016;2016:1298601.

22. Otton R, Soriano FG, Verlengia R, Curi R. Diabetes induces apoptosis in lymphocytes. J Endocrinol 2004;182:145-56.

23. Adaikalakoteswari A, Rema M, Mohan V, Balasubramanyam M. Oxidative DNA damage and augmentation of poly(ADP-ribose) polymerase/nuclear factor-kappa B signaling in patients with type 2 diabetes and microangiopathy. Int J Biochem Cell Biol 2007;39:1673-84.

24. Shiny A, Bibin YS, Shanthirani CS, Regin BS, Anjana RM, Balasubramanyam M, et al. Association of neutrophil-lymphocyte ratio with glucose intolerance: an indicator of systemic inflammation in patients 
with type 2 diabetes. Diabetes Technol Ther 2014;16:524-30.

25. Sefil F, Ulutas KT, Dokuyucu R, Sumbul AT, Yengil E, Yagiz AE, et al. Investigation of neutrophil lymphocyte ratio and blood glucose regulation in patients with type 2 diabetes mellitus. J Int Med Res 2014;42:581-8.

26. Ohn JH, Kwak SH, Cho YM, Lim S, Jang HC, Park KS, et al. 10-year trajectory of $\beta$-cell function and insulin sensitivity in the development of type 2 diabetes: a community-based prospective cohort study. Lancet Diabetes Endocrinol 2016;4:27-34.

27. Morimoto A, Tatsumi Y, Deura K, Mizuno S, Ohno Y, Miyamatsu N, et al. Impact of impaired insulin secretion and insulin resistance on the incidence of type 2 diabetes mellitus in a Japanese population: the
Saku study. Diabetologia 2013;56:1671-9.

28. Kutlu B, Burdick D, Baxter D, Rasschaert J, Flamez D, Eizirik DL, et al. Detailed transcriptome atlas of the pancreatic beta cell. BMC Med Genomics 2009;2:3.

29. Walford GA, Green T, Neale B, Isakova T, Rotter JI, Grant SF, et al. Common genetic variants differentially influence the transition from clinically defined states of fasting glucose metabolism. Diabetologia 2012;55:331-9.

30. Go MJ, Hwang JY, Park TJ, Kim YJ, Oh JH, Kim YJ, et al. Genome-wide association study identifies two novel Loci with sex-specific effects for type 2 diabetes mellitus and glycemic traits in a Korean population. Diabetes Metab J 2014;38:375-87. 